\title{
The critical role of the ventral temporal lobe in word retrieval
}

Kathryn M. Snyder ${ }^{1,2}$, Kiefer J. Forseth ${ }^{1,2}$, Cristian Donos ${ }^{1,3}$, Patrick S. Rollo ${ }^{1,2}$, Simon Fischer-Baum ${ }^{4}$, Joshua Breier ${ }^{1,5}$, Nitin Tandon ${ }^{1,2,5^{*}}$

${ }^{1}$ Vivian L. Smith Department of Neurosurgery, McGovern Medical School at UT Health Houston, Houston, TX, USA

${ }^{2}$ Texas Institute for Restorative Neurotechnologies, The University of Texas Health Science Center at Houston, Houston, TX, USA

${ }^{3}$ Faculty of Physics, University of Bucharest, Bucharest, Romania

${ }^{4}$ Department of Psychological Sciences, Rice University, Houston, TX, USA

${ }^{5}$ Memorial Hermann Hospital, Texas Medical Center, Houston, TX, USA

*Correspondence: nitin.tandon@uth.tmc.edu 


\section{Abstract}

Deficits in word retrieval are a hallmark of a variety of neurological illnesses spanning from dementia to traumatic injuries. The role of the dominant temporal lobe in fluent naming has been characterized by lesional analyses, functional imaging, and intracranial recordings, but limitations of each of these measures preclude a clear assessment of which specific constituent of the temporal lobe is critical for naming. We studied a large cohort of patients undergoing surgical resections or laser ablations of the dominant temporal lobe for medically intractable epilepsy $(n=95)$. These techniques are exceedingly effective for seizure control but often result in language declines, particularly in confrontation naming, which can be socio-economically disabling. We used a multivariate voxel-based lesion symptom mapping analysis to localize brain regions significantly associated with visual object naming deficits. We observed that posterior inferior temporal regions, centered around the middle fusiform gyrus, were significantly associated with a decline in confrontation naming. Furthermore, we found that the posterior margin of anterior temporal lobectomies was linearly correlated to a decline in visual naming with a clinically significant decline occurring once the resection extended $6 \mathrm{~cm}$ from the anterior tip of the temporal lobe. We integrated these findings with electrocorticography during naming in a subset of this population and found that the majority of cortical regions whose resection was associated with a significant decline overlapped with regions that were functionally most active prior to articulation. Importantly, these loci coincide with the sites of susceptibility artifacts during echo-planar imaging, which explains why this region has not previously been implicated. Taken together, these data highlight the crucial contribution of the posterior ventral temporal cortex in lexical access and its important role in the pathophysiology of anomia following temporal lobe resections. Surgical strategies, including the use of laser ablation to target the medial temporal lobe as well as microsurgical approaches, should attempt to preserve this region to mitigate postoperative language deficits.

Keywords: dysnomia; intracranial EEG; language; lesion-symptom mapping; temporal lobe epilepsy

Abbreviations: ATL $=$ anterior temporal lobectomy; BGA $=$ broadband gamma activity; $\mathrm{BNT}=\mathrm{Boston}$ Naming Test; BTLA = basal temporal language area; ECoG = electrocorticography; ITG = inferior temporal gyrus; $\mathrm{MTG}=$ middle temporal gyrus; $\mathrm{PHG}=$ parahippocampal gyrus; $\mathrm{pVTC}=$ posterior ventral temporal cortex; SB-MEMA = surface-based mixed effects multilevel analysis; STG = superior temporal gyrus; $\mathrm{SVR}=$ support vector regression; VLSM = voxel-based lesion symptom mapping 


\section{Introduction}

Lexical retrieval is the process of extracting a specific phonological form from a stored lexical concept. The lexicon is a theoretical construct, conceived as a hub connecting semantic systems to language-related systems such as phonology and orthography. ${ }^{1,2}$ Disruptions in lexical retrieval result in the "tip-of-thetongue" (TOT) phenomenon, or failure to retrieve a familiar word with partial recall of features associated with the target word. ${ }^{3}$ While the TOT state occurs occasionally in healthy individuals, it is a hallmark of pervasive anomia following a variety of different brain injuries. ${ }^{4}$

Anomia is particularly prevalent in patients with left language-dominant temporal lobe epilepsy, and temporal lobe resections for seizure control further increase the risk of these naming deficits. $5,6,7,8,9$ However, the precise substrates responsible for this cognitive loss are unclear. In clinical literature, a prominent focus has been on preserving the superior temporal lobe to prevent dysnomia. ${ }^{10}$ As such, a clear understanding of the most critical constituents of lexical retrieval might influence the design of surgical strategies to minimize language declines.

A variety of different methods have previously been used to isolate brain regions essential to lexical retrieval, including lesion deficit mapping, ${ }^{11,12}$ functional imaging, ${ }^{13,14,15,16,17,18,19}$ and non-invasive EEG. ${ }^{20}$ Voxel-based lesion symptom mapping (VLSM) is used to localize brain function on a voxel-by-voxel level and demonstrates the relationship between damage at any given voxel and performance on a behavioral task related to a particular cognitive function. ${ }^{21}$ Traditionally, VLSM analyses use a massunivariate approach, where the lesion-deficit relationship is determined one voxel at a time. However, this approach fails to incorporate information regarding the spatial relationship between voxels, which assumes that voxels are statistically independent and subsequently leads to a loss of statistical power and a potential bias in localization of significant regions. Using a multivariate VLSM approach mitigates these errors by modeling contributions of multiple voxels simultaneously. ${ }^{22}$ Furthermore, VLSM analyses are confounded by the fact that data are typically collected only after the occurrence of the lesion. Thus, a dataset in which both preoperative and postoperative performance measurements as well as imaging are obtained and in whom controlled lesions are produced are of particular value in evaluating the role of a given region. 
We applied multivariate VLSM analysis to a large cohort of patients who underwent surgery in the dominant left temporal lobe for treatment of drug-resistant epilepsy to specifically evaluate which component of the temporal lobe is most critical for naming. We quantified the association between each component of the temporal lobe and the change in picture naming performance to isolate brain regions associated with a decline in visual naming ability. Additionally, we integrated these results with electrocorticography $(\mathrm{ECoG})$ recordings during picture naming performed in a subset of patients who underwent intracranial electrode implantation to localize epileptic foci. This allowed us to co-localize and correlate lesion-deficit findings with brain activity.

\section{Material and Methods}

\section{Study Population}

One hundred and eighty-nine patients (105 females, 5-73 years) underwent surgical resection or ablation in the left temporal lobe for drug-resistant focal epilepsy. Study design was approved by the University of Texas Health Science Center's committee for the protection of human subjects. Of these, subjects younger than 16 years were excluded $(n=7)$. Left-hemispheric language dominance was confirmed in 146 patients by intra-carotid sodium amytal injection, ${ }^{23}$ fMRI laterality index,${ }^{24,25}$ or direct cortical stimulation. ${ }^{10}$ Patients with right $(n=15)$, bilateral $(n=3)$, or inconclusive $(n=25)$ language dominance were excluded. Additionally, subjects who were not fluent English speakers ( $\mathrm{n}=3)$, who had an IQ below 67 ( $\mathrm{n}=4)$, or who had large structural abnormalities or previous temporal lobe surgeries $(n=11)$ were excluded. Of the remaining 122 patients, 95 patients (53 females, 17-73 years, mean FSIQ 97 +/- 15) underwent neuropsychological testing and MRI prior to and following surgery. The other 27 subjects did not have all data points and were excluded. Neuropsychological testing sessions included the full Boston Naming Test (BNT), which consists of 60 black and white line drawings of objects that subjects must name within 20 s. ${ }^{26}$ Seizure outcomes were reported one year following surgery using the International League Against Epilepsy (ILAE) scoring system with scores of $1(n=57), 2(n=6), 3(n=17), 4(n=13)$, or $5(n=2)$.

\section{MRI Acquisition}

MRI scans were obtained prior to and following surgery. Preoperative scans were obtained using a 3 T whole-body magnetic resonance scanner (Philips Medical Systems) fitted with a 16-channel SENSE head coil. Images were collected using a magnetization-prepared $180^{\circ}$ radio-frequency pulse and rapid gradient- 
echo sequence with $1 \mathrm{~mm}$ sagittal slices and an in-plane resolution of $1 \mathrm{~mm}$ isotropic. Postoperative scans were acquired with the same parameters as preoperative scans. Pial surface reconstructions were computed with FreeSurfer (v6.0) ${ }^{27}$ and imported into AFNI (https://afni.nimh.nih.gov). ${ }^{28}$

Functional MRI was acquired in 35 of these patients (19 females, 19-73 years). Functional images were obtained using a gradient-recalled echo-planar imaging sequence with 33 axial slices of $3 \mathrm{~mm}$ thickness and in-plane resolution of $2.75 \mathrm{~mm} \times 2.75 \mathrm{~mm}\left(\mathrm{TE}=30 \mathrm{~ms}, \mathrm{TR}=2,015 \mathrm{~ms}\right.$, flip angle $\left.=90^{\circ}\right)$. Stimuli were presented in a block design with $20 \mathrm{~s}$ of a picture naming task alternating with $14 \mathrm{~s}$ of scrambled images as the control. Analysis of fMRI data was done using AFNI. Preprocessing included registration to the preoperative anatomical MRI and compensation for slice acquisition-dependent time shifts per volume. The temporal signal to noise ratio (tSNR) was computed for each voxel across the time series as the absolute value of the mean signal divided by its standard deviation and averaged across subjects.

\section{Voxel-Based Lesion-Symptom Mapping}

Surgical lesions were manually labeled on postoperative MRI scans in AFNI. Lesion masks were then morphed to a normative space (MNI152) by first aligning anatomical postoperative scans to preoperative scans within each subject and subsequently warping preoperative scans to a template in MNI space using nonlinear registration in Advanced Normalization Tools Software (http://stnava.github.io/ANTs/). Once in standard space, lesions masks were verified and edited by an experienced neurosurgeon.

For the VLSM analysis, only voxels that were included in the lesion masks of at least 5 subjects were analyzed. In order to reflect relative changes in BNT scores following surgery, preoperative scores were regressed out of postoperative scores using an ordinary least squares linear regression model, and the corrected postoperative BNT scores were used as the behavioral measure of interest. A linear regression was used to correct postoperative scores as there was a linear relationship between preoperative and postoperative scores. Additionally, linear regression models are more robust and enable corrected scores to maintain a gaussian distribution. ${ }^{29}$ VLSM was implemented using a multivariate support vector regression (SVR) model in order to correlate the pattern of lesioned voxels to observed deficits using a multivariate lesion symptom mapping toolbox (https://github.com/atdemarco/svrlsmgui.git). ${ }^{22}$ The SVR model was implemented using an $\varepsilon$-insensitive SVM algorithm with a radial basis function kernel from the Statistics and Machine Learning Toolbox in MATLAB. Hyperparameters (cost, $\sigma$, and $\varepsilon$ ) were 
optimized using a Baysian optimization approach to minimize resubstitution loss. Permutation-based cluster level correction of resulting beta maps was done with 5,000 permutations and a p-value of 0.05 for voxel-level and subsequent cluster-level correction in order to correct for multiple comparisons.

Resection volume was not included as a covariate in this analysis. Given that resections were constrained to the left temporal lobe, there was less spatial variability across resections. Additionally, anterior temporal resections across patients differed primarily in the extent of the posterior margin of resection, which directly influences resection volume. Thus, in this analysis, removing the effects of resection volume would be expected to distort the relationship between resected voxels and observed deficits.

\section{ECoG Acquisition and Analysis}

Of the 95 patients, 42 (24 females, 18-60 years) underwent intracranial EEG to localize seizure foci using subdural grid electrodes or depth stereo-electroencephalographic electrodes prior to resection. Subjects performed a picture naming task, in which they were presented with black and white line drawings of common objects and asked to name the object shown. ${ }^{26,30}$ Continuous audio recordings of each patient were carried out, and articulation times for each trial were manually labeled. ECoG data was collected with a sampling rate of 2,000 Hz and a bandwidth of 0.1-700 Hz using NeuroPort NSP (Blackrock Microsystems) or with a sampling rate of $1,000 \mathrm{~Hz}$ and a bandwidth of $0.15-300 \mathrm{~Hz}$ using Neurofax (Nihon Kohden). Electrode localization was performed via registration of postoperative CT with preoperative anatomical MRI scans. ${ }^{31}$ Only trials in which subjects responded correctly were used in the analysis. Analyses were done with trials time-locked to picture onset. The gamma band $(65-115 \mathrm{~Hz})$ analytic signal was extracted from the raw signal using a frequency domain bandpass Hilbert filter. ${ }^{32,33,34,35}$ Surface-based mixed-effects multilevel analysis (SB-MEMA) was used to estimate broadband gamma activity (BGA) at a population-level. ${ }^{33,34,36,37,38}$ SB-MEMA maps were calculated as a contrast in reference to the control condition to isolate regions where activity was greater for picture naming compared to scrambled images. Significance levels were computed at an alpha-level of 0.05 after using a family-wise error rate correction for multiple comparisons. VLSM results were colocalized to a standardized cortical surface in order to compute overlap between the cluster found in VLSM and SB-MEMA results. Additionally, electrodes were indexed to the closest node on the same standardized surface, and electrodes located within the region of overlap between the VLSM cluster and the thresholded SB-MEMA map were used to calculate group estimates of BGA traces. 


\section{Results}

\section{Resections}

Anterior temporal lobectomy with amygdalohippocampectomy $(\mathrm{ATL}+\mathrm{AH})$ resections $(\mathrm{n}=36)$ included the majority of the hippocampus, amygdala, temporal pole, and parahippocampal gyrus (PHG) as well as variable portions of the fusiform gyrus, the inferior temporal gyrus (ITG), the middle temporal gyrus (MTG), and the superior temporal gyrus (STG). Anterior temporal lobectomy (ATL) resections (n=9) were mainly confined to neocortical regions, which included the majority of the temporal pole and variable portions of the fusiform gyrus, ITG, MTG, and STG with relative sparing of the hippocampus, amygdala, and PHG. Additionally, 4 patients underwent modified anterior lobectomies. One patient underwent an ATL with amygdalotomy and relative sparing of the hippocampus. One patient underwent an ATL+AH with additional resection of portions of the insula. One patient underwent an ATL+AH with additional resection of a portion of the mesial frontal region. One patient underwent an ATL with additional resection of a portion of the basal frontal lobe. Resection overlap of all subject who underwent ATL procedures is shown in Supplementary Fig 1a. Laser interstitial thermal ablations (LITT) of the mesial temporal lobe $(n=31)$ were confined to the hippocampus and amygdala and occasionally included a small portion of PHG immediately adjacent to the hippocampal region (Supplementary Fig 1b). Focal resections ( $\mathrm{n}=15$ ) were tailored based on individual clinical evaluations and were distributed across the left temporal lobe. Four patients had resections confined primarily to the temporal pole. Two patients underwent a laser ablation of a temporal periventricular nodular heterotopia (PVNH). Four patients underwent small resections located within the temporal-occipital cortex. One patient underwent a temporal mass resection involving lateral anterior temporal regions. One patient had a temporal topectomy in the lateral temporal area involving mostly MTG. One patient had a resection that included a portion of the anterior STG. One patient had a small resection located within posterior STG. One patient underwent an extensive ATL that included larger portions of the fusiform gyrus, ITG, and MTG. Resection overlap across all patients is shown in Fig 1. A threshold was applied to the resection overlap map to show only voxels resected in at least 5 patients, which reflects the voxels included in the VLSM analysis.

\section{BNT Scores}

The average raw preoperative BNT score was $45.35+/-8.85$, and the average raw postoperative BNT score was 41.75 +/- 10.30 (Fig 2a). Prior to fitting the SVR model, the preoperative BNT score was 
regressed out of the postoperative BNT score using a linear regression. The resulting corrected postoperative BNT scores were represented as a standardized percentage centered at $0 \%$, which corresponded to no change between preoperative and postoperative scores (Fig 2b). These standardized percentage scores can be interpretated as a single score representation of BNT performance following surgery relative to their preoperative performance. No change in BNT performance corresponded to $0 \%$ (grey dotted line in Fig 2b), a 5-point increase in BNT performance following surgery (the reliable change index for significant improvement) corresponded to $10.57 \%$ (green dotted line in Fig 2b), and a 4-point decrease in BNT performance (the reliable change index for significant decline) corresponded to $-8.46 \%$ (red dotted line in Fig 2b). ${ }^{39}$ Fig 2c shows the correlation between preoperative and postoperative BNT scores both before and after linear regression. Scatter points are colored as clinically significant improvement (green), clinically significant decline (red), or clinically insignificant change (grey). The relationship between corrected postoperative BNT scores and the difference between postoperative and preoperative BNT scores is shown in Supplementary Fig 2.

Statistical analyses were performed to examine the effect of language dominance on naming decline following left temporal lobe resections using unpaired, two-sample t-tests. In patients who underwent left ATL procedures, the change in BNT performance following surgery was significantly different in left language dominant patients compared to right language dominant patients (two-tailed $\mathrm{p}<0.001$ ) with left language dominant patients exhibiting a greater decline following resection (one-tailed $\mathrm{p}<0.001$ ). In patients who underwent left LITT procedures, there was no difference in the change in BNT performance following surgery between left and right language dominant patients (two-tailed $p=0.53$ ).

\section{VLSM}

Fig $3 \mathrm{a}$ and $3 \mathrm{~b}$ show the unthresholded Beta map computed after fitting the multivariate SVR model. The Beta value of a given voxel represents its contribution to BNT performance where negative values indicate a decline in BNT performance following resection of the corresponding voxel. The Beta map revealed that resection of posterior inferior temporal regions contribute more to a decline in naming compared to anterolateral temporal regions. Fig $3 \mathrm{c}$ and $3 \mathrm{~d}$ show the most significant cluster contributing to decline in BNT performance following permutation-based cluster level correction of the Beta map $(\mathrm{p}=0.0248)$. The cluster primarily included the fusiform gyrus, PHG, and ITG. It had a volume of 5,656 $\mathrm{mm}^{3}$ and a center of mass located in the fusiform gyrus ([-33.1, -12.3, -33.4] in MNI space). 
Less than $3 \%$ of voxels within the significant cluster were located within the hippocampus. To look more closely at the role of the hippocampus and its effect in naming, we computed the correlation between the percent of the hippocampus removed and the corrected postoperative BNT score $(r=-0.21, \mathrm{p}=0.044)$. We found that the percentage of the hippocampus removed was not strongly correlated with naming decline and explained less than 5\% of the variance in corrected BNT scores (Supplementary Fig 3).

To investigate the nature of the relationship between the posterior extent of ATL resections and decline in BNT performance, we computed the correlation between the distance from the tip of the temporal pole to the most posterior coordinate of the resection mask and the corrected postoperative BNT score across subjects who underwent standard ATL or temporal pole resections (fig 4). We found that the extent of the posterior margin of the resection was linearly correlated to a decline in postoperative naming $(r=-0.58, p$ $<0.001)$, with a significant decline in BNT performance $(\mathrm{RCI}=-4)$ occurring once the posterior margin reached $\sim 6 \mathrm{~cm}$ from the tip of the temporal lobe.

The average tSNR values per voxel of fMRI scans acquired during a picture naming task were assessed in a subset of patients from our cohort (fig 5). Regions with the lowest tSNR included the orbitofrontal cortex and the ventral temporal cortex. Whole brain tSNR ranged from 2.35 to 131.5 with an average of 79.40 +/- 21.73. The mean tSNR of the significant cluster computed using VLSM was 51.93 +/- 21.29.

\section{ECoG Analysis}

The average reaction time for picture naming was $1,355 \mathrm{~ms}$ with a mean accuracy of $93.65+/-0.04 \%$. Fig 6a shows the electrode coverage across patients with ECoG included in the analysis. SB-MEMA analysis during picture naming showed peak BGA spreading anteriorly along the ventral temporal stream 500 to $750 \mathrm{~ms}$ following picture onset, and regions with greater cortical responses to picture naming compared to scrambled images were located primarily within the posterior ventral temporal lobe (fig 6b). This is concordant with prior work revealing a semantic-specific increase in activity in this region using both fMRI and ECoG. ${ }^{40}$ The thresholded SB-MEMA map overlapped with $78.8 \%$ of the significant cluster computed using VLSM (fig 6c). Overlap was primarily seen in the fusiform gyrus. Fig 6d-e shows the average percent change in BGA relative to picture onset during pictures (purple) and scrambled images (black) for electrodes located within the overlap between the SB-MEMA map and the VLSM cluster with 
an absolute BGA change of at least 25\%. There was a significant increase in BGA compared to the control condition (scrambled images) beginning around $250 \mathrm{~ms}$ following picture onset with a peak increase occurring within 500 to $750 \mathrm{~ms}$. Significance was computed using an unpaired t-test with a false discovery rate (FDR) corrected p-value of 0.05 .

\section{Discussion}

We isolated the critical cortical constituents for confrontation naming using multivariate VLSM and integrated these findings with ECoG within the same patient population to derive quantifiable measures of convergence between lesion-deficit localization and brain activity. We found that damage to the dominant posterior ventral temporal cortex (pVTC) is significantly associated with pervasive deficits in visual naming. Together, these data firmly establish the role of the fusiform gyrus as a critical semantic access hub that is essential for lexical retrieval..$^{40,41,42}$

Previous studies using cortical stimulation mapping have highlighted the importance of pVTC, also referred to as the basal temporal language area (BTLA), in confrontation naming. ${ }^{40,43}$ More specifically, it has been shown that disruption of the fusiform gyrus, PHG, and ITG results in speech arrest. ${ }^{43,44}$ Additionally, Forseth et al. ${ }^{40}$ observed that stimulation of the fusiform gyrus specifically disrupted object naming for both pictures and auditory descriptions without disruption of sentence repetition or sensorimotor effects, which implicates its role in semantic processing as opposed to audio-visual integration. Despite clear evidence for acute disruption in language function following electrical stimulation, previous studies have underplayed the role of this region in chronic lesions, reporting that resection of the BTLA results in no significant language impairments or only transient aphasias with the majority of language deficits resolving within one month following surgery. ${ }^{43,45,46}$ It has been hypothesized that these findings might suggest that the BTLA does not play an important role in language $\mathrm{e}^{47,48,49}$ or that its role within language networks is not essential. ${ }^{49,50}$ However, other studies have found BTLA resections to be associated with more pervasive aphasias. ${ }^{16,51,52}$ Our data show that resections involving the left dominant BTLA do result in long-term language deficits with many patients reporting noticeable word finding difficulties at the time of postoperative testing, which took place at least 3 months following surgery. Furthermore, we found that left language dominant patients experienced significantly greater declines in naming ability compared to right language dominant patients following left ATL 
procedures, indicating that the function of the dominant BTLA is distinct from that of the nondominant BTLA.

Despite accumulating evidence implicating pVTC in semantic processing, functional neuroimaging studies have reported variable results regarding semantic-associated activations in the ventral temporal lobe, and many have localized semantic function to more lateral temporal regions. ${ }^{53,54,55,56}$ However, limitations of echo planar imaging may restrict the ability to study the medial temporal lobe using fMRI. Due to its proximity to both the sinuses and the skull, this area is particularly affected by image distortion and signal loss caused by susceptibility-induced magnetic field gradients, and efforts to minimize these artifacts in order to improve accuracy often result in a reduced SNR. ${ }^{57,58} \mathrm{We}$ found that ventral temporal regions, especially those identified as significant by our VLSM analysis, were associated with a low SNR on fMRI, which may explain why this region has been under-appreciated as the locus responsible for postoperative deficits.

The exact role of the hippocampus in naming is not clearly understood. It has been shown that laser ablation of the hippocampus and amygdala reduces the risk of postoperative naming impairment in comparison to standard ATL procedures. ${ }^{52,59,60,61}$ Concordantly, we did not find a significant association between the hippocampus and naming performance, and less than $3 \%$ of voxels included in the significant cluster belonged to the hippocampus. Additionally, the percentage of the hippocampus removed or ablated was not significantly correlated with a change in BNT performance. Thus, our results do not support that the hippocampus is an essential component of object naming.

It is well-known that dominant ATL resections increase the risk of postoperative naming deficits. ${ }^{41,62,63,64}$ Based on these findings and studies in patients with semantic dementia, it has also been hypothesized that the temporal pole functions as the critical access hub in lexical semantic processing. ${ }^{65,66}$ Our results show that resection of the temporal pole within $\sim 3 \mathrm{~cm}$ of the anterior tip of the temporal lobe is not significantly associated with postoperative naming deficits. However, we found that the posterior margin of ATL resections was linearly correlated with postoperative naming scores, with resections extending more posteriorly resulting in larger naming declines and a clinically significant decline occurring if the resection extended more than $6 \mathrm{~cm}$ from the temporal pole. Trimmel et al. ${ }^{67}$ found the left $\mathrm{pVTC}$ to be functionally connected to the left anterior STG and temporal pole, and it has been suggested that naming decline 
following dominant ATL resections may be the result of partial disconnections between the resected regions and pVTC. ${ }^{19,67}$ While more anterior regions may support other aspects of semantic processing, our data does not support that these regions are essential to lexical retrieval. As such, language declines following resection of more anterior portions of the left temporal lobe may be the result of increased disruption of ventral temporal connections following ATL resections as opposed to removal of critical lexical semantic processing regions, and observed visual naming decline may reflect the proportion of disruption to the antero-posterior-basal temporal language network.

Using both multivariate VLSM and ECoG, we have demonstrated the essential contribution of pVTC, specifically the middle fusiform cortex, to lexical access. We found that posterior inferior temporal regions, centered around the fusiform gyrus, were significantly associated with a decline in visual object naming ability following resection, and the majority of these regions were most active on ECoG during picture naming just prior to articulation. These findings build on our prior work ${ }^{40}$ as well as a recent lesiondeficit mapping study ${ }^{52}$ elucidating the role of the fusiform gyrus in naming. Altogether, these results support the importance of pVTC in the lexical semantic network, and further implicate the fusiform gyrus as a critical lexical sematic access hub.

\section{Acknowledgements}

We thank all the patients who participated in this study, laboratory members at the Tandon lab (Oscar Woolnough and Jessica Johnson), neurologists at the Texas Comprehensive Epilepsy Program who participated in the care of these patients, and all the nurses and technicians in the Epilepsy Monitoring Unit at Memorial Hermann Hospital who helped make this research possible.

\section{Funding}

This work was supported by the National Institute for Deafness and Other Communication Disorders DC014589.

\section{Competing Interests}

The authors report no competing interests. 


\section{References}

1. Indefrey P, Levelt WJM. The spatial and temporal signatures of word production components. Cognition. 2004; 92(1):101-144. doi:10.1016/j.cognition.2002.06.001

2. Friedmann N, Biran M, Dotan D. Lexical retrieval and its breakdown in aphasia and developmental language impairment. In: Boeckx C, Grohmann KK, eds. The Cambridge Handbook of Biolinguistics. Cambridge Handbooks in Language and Linguistics. Cambridge University Press; 2013:350-374. doi:10.1017/CBO9780511980435.021

3. Brown R, McNeill D. The "tip of the tongue" phenomenon. Journal of Verbal Learning and Verbal Behavior. 1966; 5(4):325-337. doi:10.1016/S0022-5371(66)80040-3

4. Margolin DI, Pate DS, Friedrich FJ, Elia E. Dysnomia in dementia and in stroke patients: Different underlying cognitive deficits. Journal of Clinical and Experimental Neuropsychology. 1990; 12(4):597-612. doi:10.1080/01688639008401004

5. Mayeux R, Brandt J, Rosen J, Benson D. Interictal memory and language impairment in temporal lobe epilepsy. Neurology. 1980; 30:120-120.

6. Bell BD, Davies KG. Anterior Temporal Lobectomy, Hippocampal Sclerosis, and Memory: Recent Neuropsychological Findings. Neuropsychology Review. 1998; 8(1):25-41. doi:10.1023/A:1025679122911

7. Schwarz M, Pauli E, Stefan H. Model based prognosis of postoperative object naming in left temporal lobe epilepsy. Seizure - European Journal of Epilepsy. 2005; 14(8):562-568. doi:10.1016/j.seizure.2005.09.001

8. Drane DL, Ojemann GA, Aylward E, et al. Category-specific naming and recognition deficits in temporal lobe epilepsy surgical patients. Neuropsychologia. 2008; 46(5):1242-1255. doi:10.1016/j.neuropsychologia.2007.11.034

9. Hamberger MJ. Object naming in epilepsy and epilepsy surgery. Epilepsy \& Behavior. 2015; 46:27-33. doi:10.1016/j.yebeh.2014.12.019

10. Ojemann G, Ojemann J, Lettich E, Berger M. Cortical language localization in left, dominant hemisphere: An electrical stimulation mapping investigation in 117 patients. Journal of Neurosurgery. 1989; 71(3):316-326. doi:10.3171/jns.1989.71.3.0316

11. Warrington EK. The Selective Impairment of Semantic Memory. Quarterly Journal of Experimental Psychology. 1975; 27(4):635-657. doi:10.1080/14640747508400525 
12. Nestor PJ, Fryer TD, Hodges JR. Declarative memory impairments in Alzheimer's disease and semantic dementia. NeuroImage. 2006; 30(3):1010-1020. doi:10.1016/j.neuroimage.2005.10.008

13. Damasio H, Grabowski TJ, Tranel D, Hichwa RD, Damasio AR. A neural basis for lexical retrieval. Nature. 1996; 380(6574):499-505. doi:10.1038/380499a0

14. Noppeney U, Price CJ. A PET Study of Stimulus- and Task-Induced Semantic Processing. NeuroImage. 2002; 15(4):927-935. doi:10.1006/nimg.2001.1015

15. Bright P, Moss H, Tyler LK. Unitary vs multiple semantics: PET studies of word and picture processing. Brain and Language. 2004; 89(3):417-432. doi:10.1016/j.band1.2004.01.010

16. Sharp DJ, Scott SK, Wise RJS. Retrieving meaning after temporal lobe infarction: The role of the basal language area. Annals of Neurology. 2004; 56(6):836-846. doi:10.1002/ana.20294

17. Spitsyna G, Warren JE, Scott SK, Turkheimer FE, Wise RJS. Converging language streams in the human temporal lobe. J Neurosci. 2006; 26(28):7328-7336. doi:10.1523/JNEUROSCI.0559$\underline{06.2006}$

18. Binder JR, Desai RH, Graves WW, Conant LL. Where Is the Semantic System? A Critical Review and Meta-Analysis of 120 Functional Neuroimaging Studies. Cerebral Cortex. 2009; 19(12):27672796. doi:10.1093/cercor/bhp055

19. Fonseca ATD, Guedj E, Alario FX, et al. Brain regions underlying word finding difficulties in temporal lobe epilepsy. Brain. 2009; 132(10):2772-2784. doi:10.1093/brain/awp083

20. Marinkovic K, Dhond RP, Dale AM, Glessner M, Carr V, Halgren E. Spatiotemporal dynamics of modality-specific and supramodal word processing. Neuron. 2003; 38(3):487-497. doi:10.1016/s0896-6273(03)00197-1

21. Bates E, Wilson SM, Saygin AP, et al. Voxel-based lesion-symptom mapping. Nature Neuroscience. 2003; 6(5):448-450. doi:10.1038/nn1050

22. DeMarco, Andrew T, Turkeltaub, Peter E. A multivariate lesion symptom mapping toolbox and examination of lesion-volume biases and correction methods in lesion-symptom mapping. Human Brain Mapping. 2018; 39:4169-4182. doi:10.1002/hbm.24289

23. Wada J, Rasmussen T. Intracarotid injection of sodium amytal for the lateralization of cerebral speech dominance: experimental and clinical observations. Journal of Neurosurgery JNS. 2007;106(6):1117-1133. doi: 10.3171/jns.2007.106.6.1117 
24. Ellmore TM, Beauchamp MS, Breier JI, et al. Temporal lobe white matter asymmetry and language laterality in epilepsy patients. Neuroimage. 2010; 49(3):2033-2044. doi:10.1016/j.neuroimage.2009.10.055

25. Conner CR, Ellmore TM, Pieters TA, DiSano MA, Tandon N. Variability of the Relationship between Electrophysiology and BOLD-fMRI across Cortical Regions in Humans. J Neurosci. 2011; 31(36):12855. doi:10.1523/JNEUROSCI.1457-11.2011

26. Kaplan E, Goodglass Harold, Weintraub Sandra, Goodglass Harold. Boston Naming Test. Lea \& Febiger; 1983.

27. Dale AM, Fischl B, Sereno MI. Cortical Surface-Based Analysis: I. Segmentation and Surface Reconstruction. NeuroImage. 1999; 9(2):179-194. doi:10.1006/nimg.1998.0395

28. Saad ZS, Reynolds RC. SUMA. Neuroimage. 2012; 62(2):768-773. doi:10.1016/j.neuroimage.2011.09.016

29. Bishop CM. Pattern Recognition and Machine Learning. 1st ed. Springer; 2006.

30. Snodgrass J, Vanderwart M. A standardized set of 260 pictures: norms for name agreement, image agreement, familiarity, and visual complexity. J Exp Psychol Hum Learn. 1980; 6(2):174-215. doi:10.1037//0278-7393.6.2.174

31. Pieters TA, Conner CR, Tandon N. Recursive grid partitioning on a cortical surface model: an optimized technique for the localization of implanted subdural electrodes: Clinical article. Journal of Neurosurgery JNS. 2013; 118(5):1086-1097. doi:10.3171/2013.2.JNS121450

32. Bruns A, Eckhorn R, Jokeit H, Ebner A. Amplitude envelope correlation detects coupling among incoherent brain signals. NeuroReport. 2000; 11(7).

33. Conner CR, Chen G, Pieters TA, Tandon N. Category specific spatial dissociations of parallel processes underlying visual naming. Cereb Cortex. 2014; 24(10):2741-2750. doi: $10.1093 /$ cercor/bht130

34. Kadipasaoglu CM, Forseth K, Whaley M, et al. Development of grouped icEEG for the study of cognitive processing. Front Psychol. 2015; 6:1008-1008. doi:10.3389/fpsyg.2015.01008

35. Whaley ML, Kadipasaoglu CM, Cox SJ, Tandon N. Modulation of Orthographic Decoding by Frontal Cortex. J Neurosci. 2016; 36(4):1173-1184. doi:10.1523/JNEUROSCI.2985-15.2016

36. Fischl B, Sereno MI, Tootell RB, Dale AM. High-resolution intersubject averaging and a coordinate system for the cortical surface. Hum Brain Mapp. 1999; 8(4):272-284. doi:10.1002/(sici)1097-0193(1999)8:4<272::aid-hbm10>3.0.co;2-4 
37. Chen G, Saad ZS, Nath AR, Beauchamp MS, Cox RW. FMRI group analysis combining effect estimates and their variances. Neuroimage. 2012; 60(1):747-765. doi:10.1016/j.neuroimage.2011.12.060

38. Kadipasaoglu CM, Baboyan VG, Conner CR, Chen G, Saad ZS, Tandon N. Surface-based mixed effects multilevel analysis of grouped human electrocorticography. NeuroImage. 2014; 101:215224. doi:10.1016/j.neuroimage.2014.07.006

39. Sachs BC, Lucas JA, Smith GE, et al. Reliable change on the Boston naming test. J Int Neuropsychol Soc. 2012; 18(2):375-378. doi:10.1017/S1355617711001810

40. Forseth, Kiefer J, Kadipasaoglu, Cihan M, Connor, Christopher R, Hickok, Gregory, Knight, Robert T, Tandon, Nitin. A lexical semantic hub for heteromodal naming in middle fusiform gyrus. Brain. 2018; 141:2112-2126. doi:10.1093/brain/awy120

41. Binder JR, Desai RH. The neurobiology of semantic memory. Trends Cogn Sci. 2011; 15(11):527536. doi: $10.1016 /$ j.tics.2011.10.001

42. Woolnough O, Donos C, Rollo PS, et al. Spatiotemporal dynamics of orthographic and lexical processing in the ventral visual pathway. Nature Human Behaviour. 2021; 5(3):389-398. doi:10.1038/s41562-020-00982-w

43. Luders H, Lesser RP, Hahn J, et al. Basal temporal language area. Brain. 1991; 114(2):743-754. doi:10.1093/brain/114.2.743

44. Burnstine TH, Lesser RP, Hart J, et al. Characterization of the basal temporal language area in patients with left temporal lobe epilepsy. Neurology. 1990; 40(6):966. doi:10.1212/WNL.40.6.966 45. Penfield W, Roberts L. Speech and Brain Mechanisms. Princeton University Press; 1959.

46. Loring DW, Meador KJ, Lee GP. Effects of temporal lobectomy on generative fluency and other language functions. Archives of Clinical Neuropsychology. 1994; 9(3):229-238. doi:10.1093/arclin/9.3.229

47. Duffau H, Capelle L, Denvil D, et al. Usefulness of intraoperative electrical subcortical mapping during surgery for low-grade gliomas located within eloquent brain regions: functional results in a consecutive series of 103 patients. Journal of Neurosurgery. 2003; 98(4):764-778. doi: 10.3171/jns.2003.98.4.0764

48. Lambon Ralph MA, Cipolotti L, Manes F, Patterson K. Taking both sides: do unilateral anterior temporal lobe lesions disrupt semantic memory? Brain. 2010; 133(11):3243-3255. doi: $10.1093 /$ brain/awq264 
49. Wilson SM, Lam D, Babiak MC, et al. Transient aphasias after left hemisphere resective surgery. Journal of Neurosurgery JNS. 2015; 123(3):581-593. doi:10.3171/2015.4.JNS141962

50. Krauss GL, Fisher R, Plate C, et al. Cognitive Effects of Resecting Basal Temporal Language Areas. Epilepsia. 1996; 37(5):476-483. doi:10.1111/j.1528-1157.1996.tb00594.x

51. Langfitt JT, Rausch R. Word-Finding Deficits Persist After Left Anterotemporal Lobectomy. Archives of Neurology. 1996; 53(1):72-76. doi:10.1001/archneur.1996.00550010090021

52. Binder, Jeffrey R. Temporal lobe regions essential for preserved picture naming after temporal epilepsy surgery. Epilepsia. 2020; 00:1-10. doi:10.1111/epi.16643

53. Thompson-Schill SL. Neuroimaging studies of semantic memory: inferring "how" from "where." Neuropsychologia. 2003;41(3):280-292. doi:10.1016/S0028-3932(02)00161-6

54. Catani M, ffytche DH. The rises and falls of disconnection syndromes. Brain. 2005;128(10):22242239. doi:10.1093/brain/awh622

55. Martin A. The Representation of Object Concepts in the Brain. Annu Rev Psychol. 2007;58(1):2545. doi:10.1146/annurev.psych.57.102904.190143

56. Ralph MAL, Jefferies E, Patterson K, Rogers TT. The neural and computational bases of semantic cognition. Nature Reviews Neuroscience. 2017;18(1):42-55. doi:10.1038/nrn.2016.150

57. Ojemann JG, Akbudak E, Snyder AZ, McKinstry RC, Raichle ME, Conturo TE. Anatomic Localization and Quantitative Analysis of Gradient Refocused Echo-Planar fMRI Susceptibility Artifacts. NeuroImage. 1997;6(3):156-167. doi:10.1006/nimg.1997.0289

58. Olman CA, Davachi L, Inati S. Distortion and signal loss in medial temporal lobe. PLoS One. 2009; 4(12):e8160-e8160. doi:10.1371/journal.pone.0008160

59. Drane DL, Loring DW, Voets NL, et al. Better object recognition and naming outcome with MRIguided stereotactic laser amygdalohippocampotomy for temporal lobe epilepsy. Epilepsia. 2015; 56(1):101-113. doi:10.1111/epi.12860

60. Hoppe C, Witt JA, Helmstaedter C, Gasser T, Vatter H, Elger CE. Laser interstitial thermotherapy (LiTT) in epilepsy surgery. Seizure - European Journal of Epilepsy. 2017; 48:45-52. doi:10.1016/j.seizure.2017.04.002

61. Donos, Cristian, Breier, Joshua, Friedman, Elliot, et al. Laser ablation for mesial temporal lobe epilepsy: Surgical and cognitive outcomes with and without mesial temporal sclerosis. Epilepsia. 2018; 59:1421-1432. doi:10.1111/epi.14443 
62. Sabsevitz DS, Swanson SJ, Hammeke TA, et al. Use of preoperative functional neuroimaging to predict language deficits from epilepsy surgery. Neurology. 2003; 60(11):1788. doi:10.1212/01.WNL.0000068022.05644.01

63. Goucha T, Friederici AD. The language skeleton after dissecting meaning: A functional segregation within Broca's Area. NeuroImage. 2015; 114:294-302. doi:10.1016/j.neuroimage.2015.04.011

64. Middlebrooks CD, Kerr T, Castel AD. Selectively Distracted: Divided Attention and Memory for Important Information. Psychol Sci. 2017; 28(8):1103-1115. doi:10.1177/0956797617702502

65. Patterson K, Nestor PJ, Rogers TT. Where do you know what you know? The representation of semantic knowledge in the human brain. Nature Reviews Neuroscience. 2007; 8(12):976-987. doi: $10.1038 / \mathrm{nrn} 2277$

66. Lambon Ralph MA. Neurocognitive insights on conceptual knowledge and its breakdown. Philos Trans R Soc Lond B Biol Sci. 2013; 369(1634):20120392-20120392. doi:10.1098/rstb.2012.0392

67. Trimmel K, van Graan AL, Caciagli L, et al. Left temporal lobe language network connectivity in temporal lobe epilepsy. Brain. 2018; 141(8):2406-2418. doi:10.1093/brain/awy164 


\section{Figures}

A

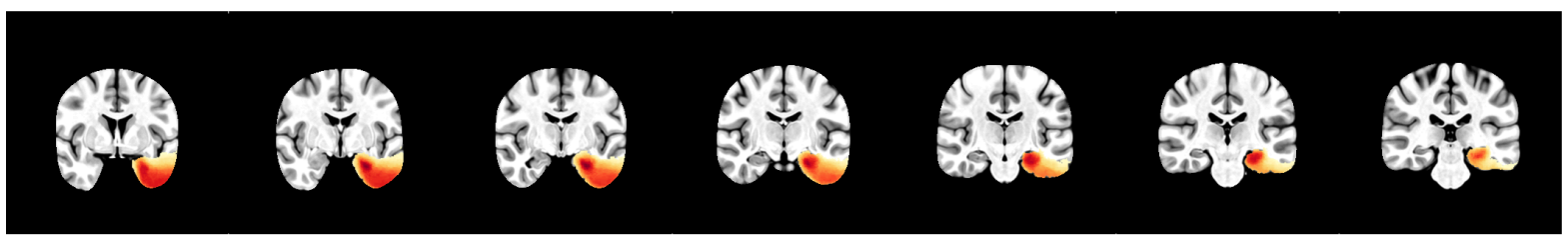

B
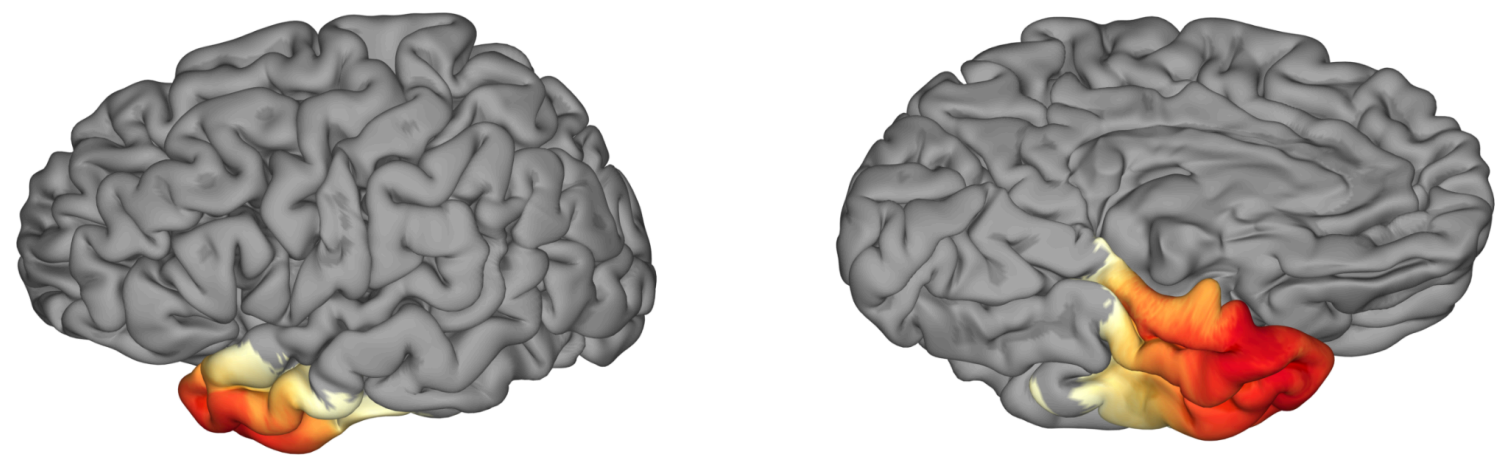

5

67

\# patients

Figure 1. Lesion Overlap. The resection mask coverage across all subjects shown in the volume (A) and on the cortical surface (B) with a threshold applied to show only voxels that were included in the resections of at least 5 subjects. 

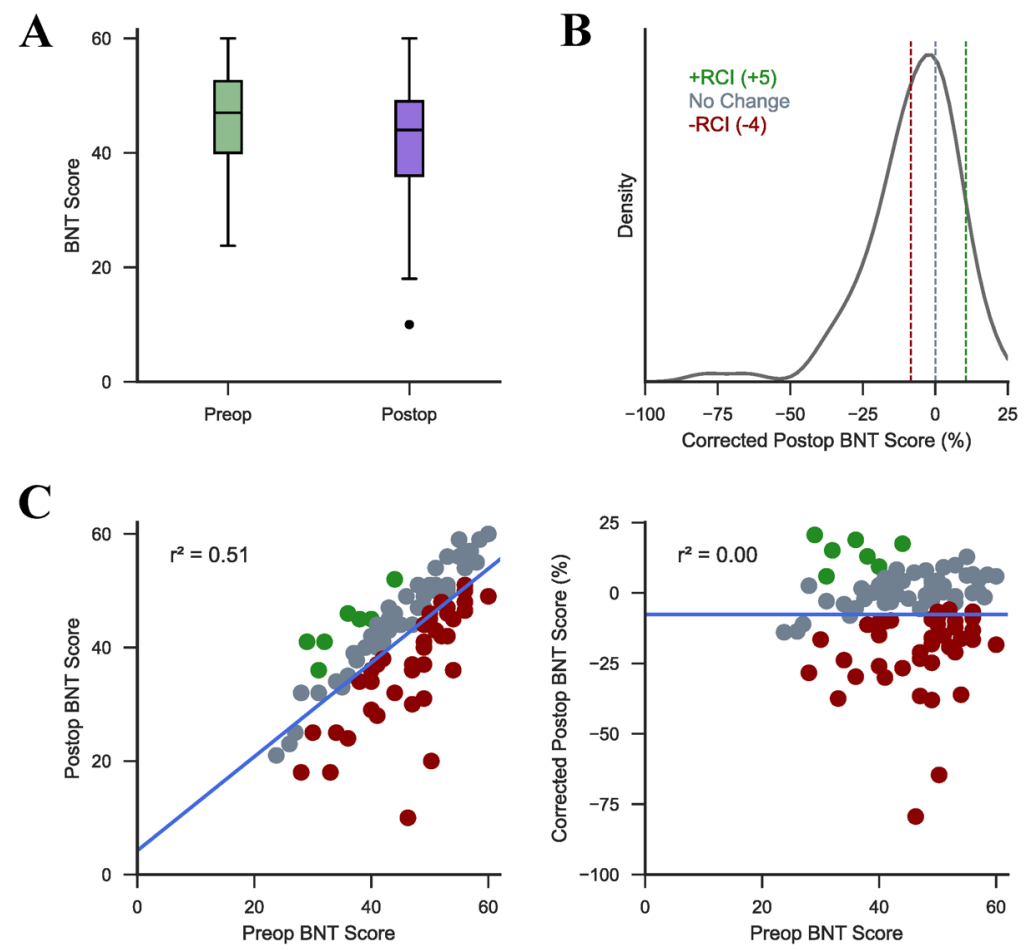

Figure 2. SVR Covariates. (A) The distribution of raw preoperative and postoperative BNT scores across all subjects. (B) The distribution of postoperative BNT scores following regression of preoperative BNT scores displayed as a standardized percentage. Dotted lines indicate corrected scores corresponding to a difference between postoperative and preoperative BNT scores of 0 (no change; grey), +5 (+RCI; green), and -4 (-RCI; red). (C) The correlation between preoperative and postoperative BNT scores (displayed as the percentage of correct answers) before (left) and after (right) regression. Scatter points are colored as clinically significant improvement (green), clinically significant decline (red), or clinically insignificant change (grey). 
A

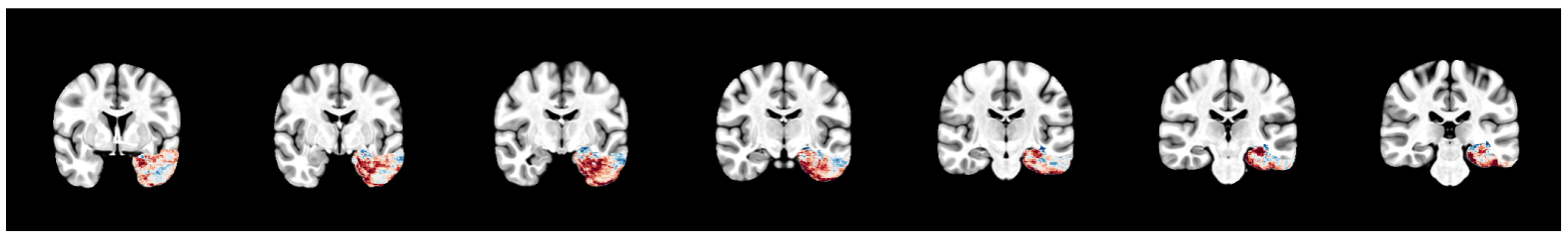

B
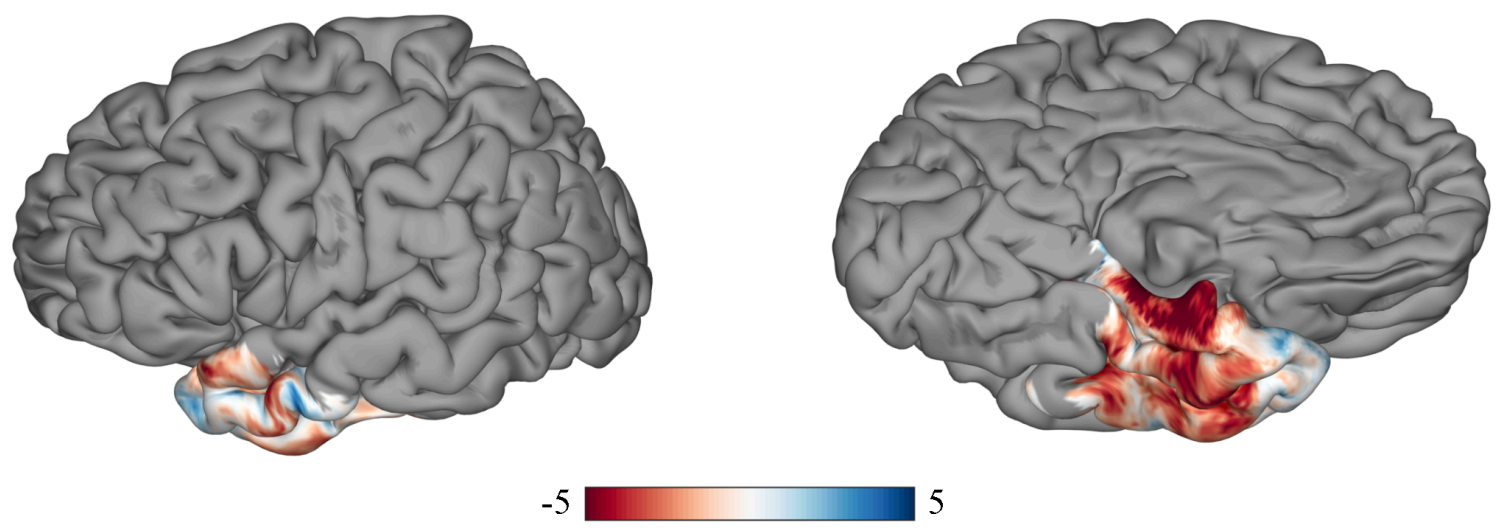

Beta value

C

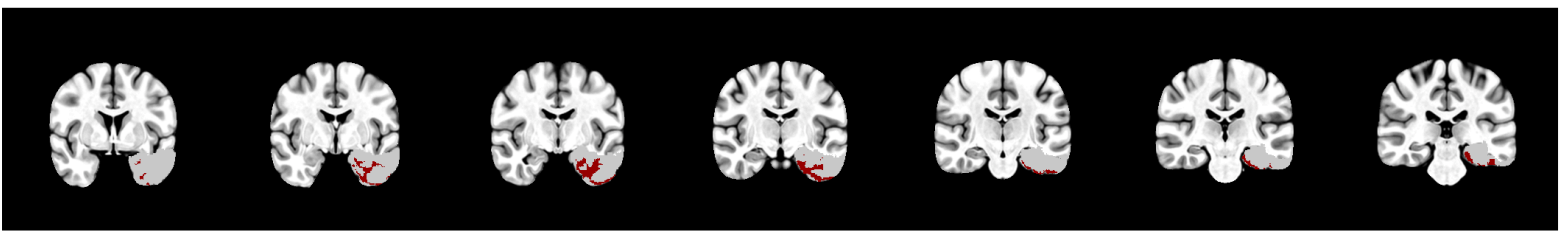

D
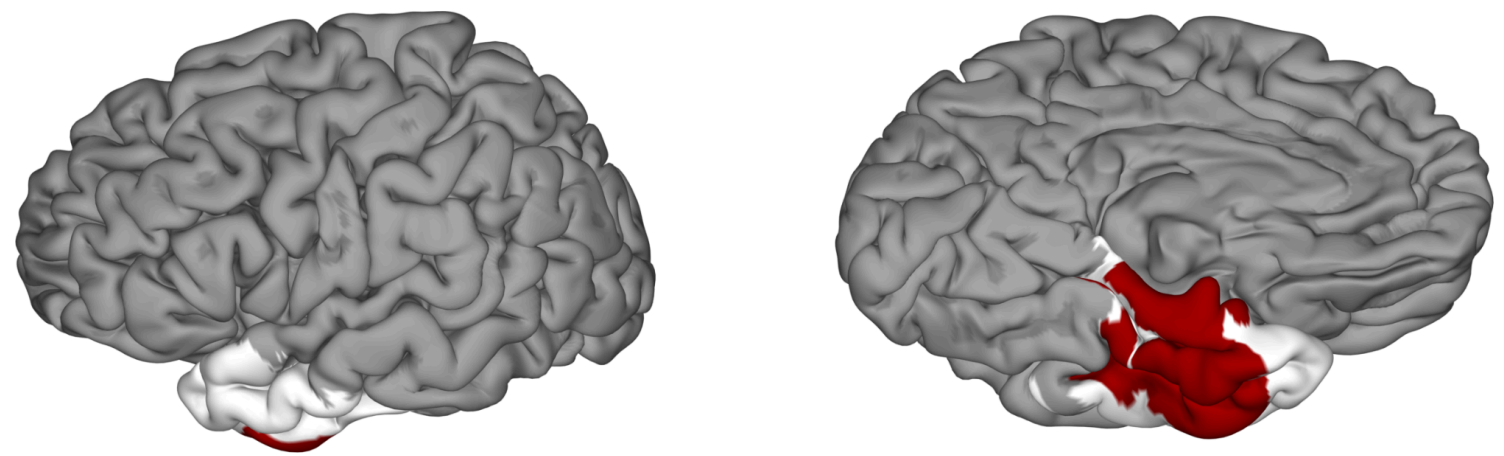

Figure 3. VLSM Results. (A-B) The Beta map computed using a multivariate SVR model shown in the volume (A) and on the cortical surface (B). More negative values indicate that resection of the given voxel results in decline in BNT performance. (C-D) The most significant cluster following permutation-based cluster level correction of the Beta map shown in the volume (C) and on the cortical surface (D). Regions included in the cluster are shown in red, and regions not included in the cluster but included in the analysis are shown in white. 

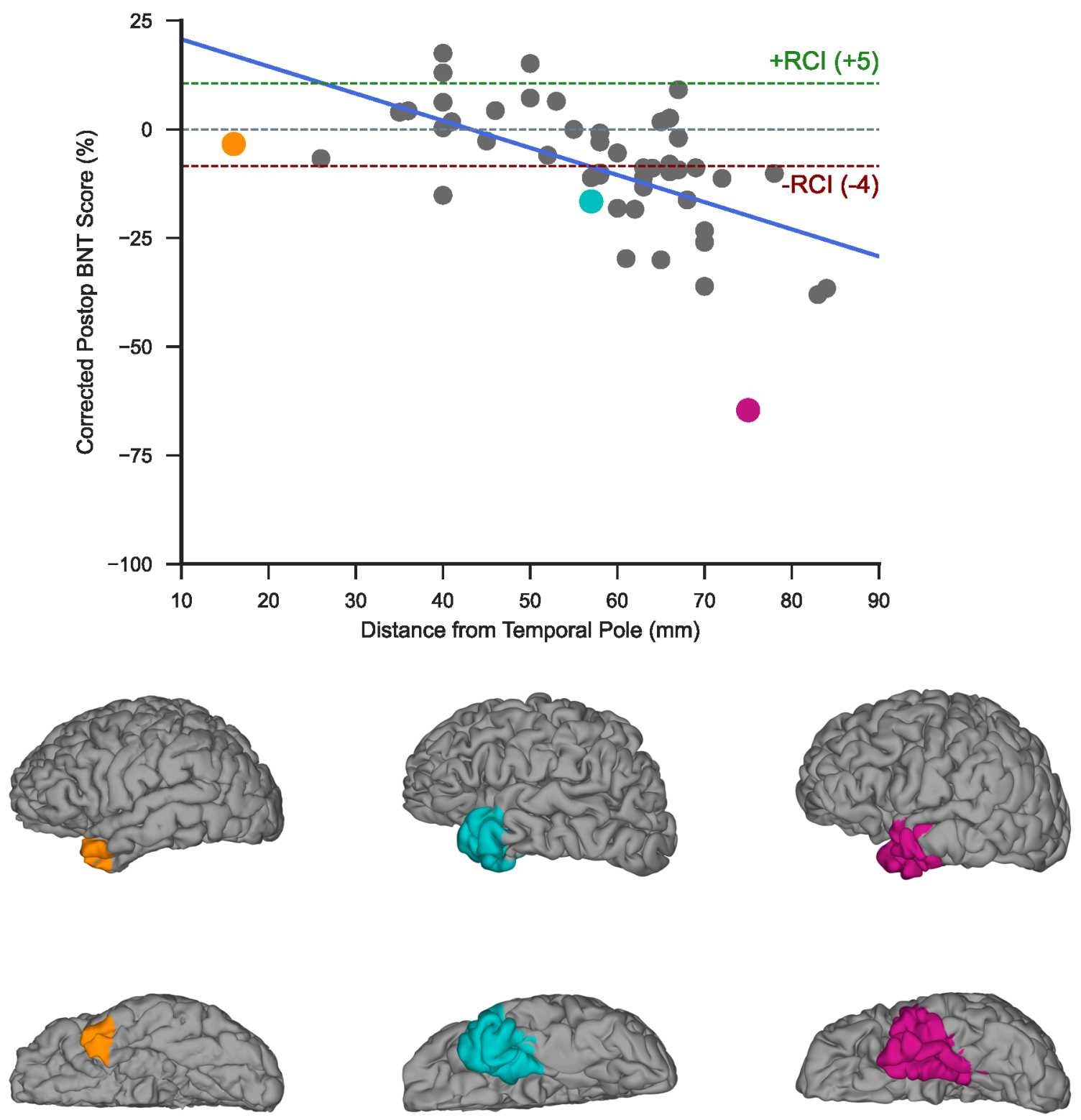

Figure 4. Analysis of Posterior Extent of ATL Resections. The correlation between corrected BNT score and the posterior margin of ATL resections measured as distance from the tip of the temporal pole $(\mathrm{r}=-0.58, \mathrm{p}<0.001)$. The resections of three examples patients are shown on the cortical surface and highlighted in the scatter plot in the corresponding color. 
A

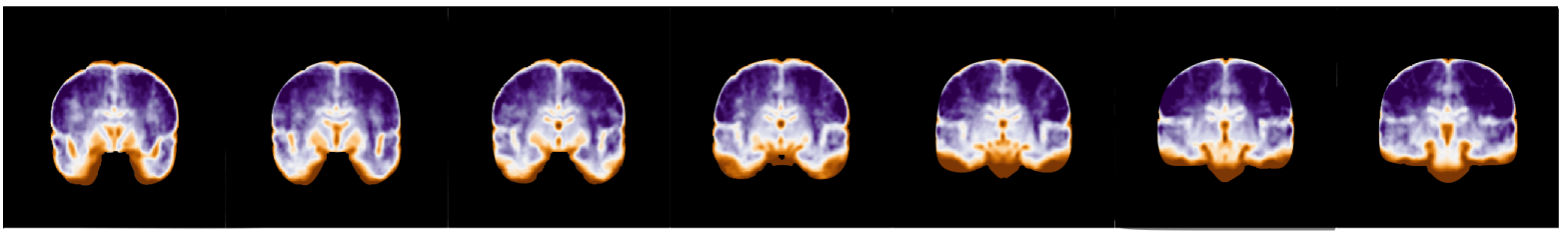

B
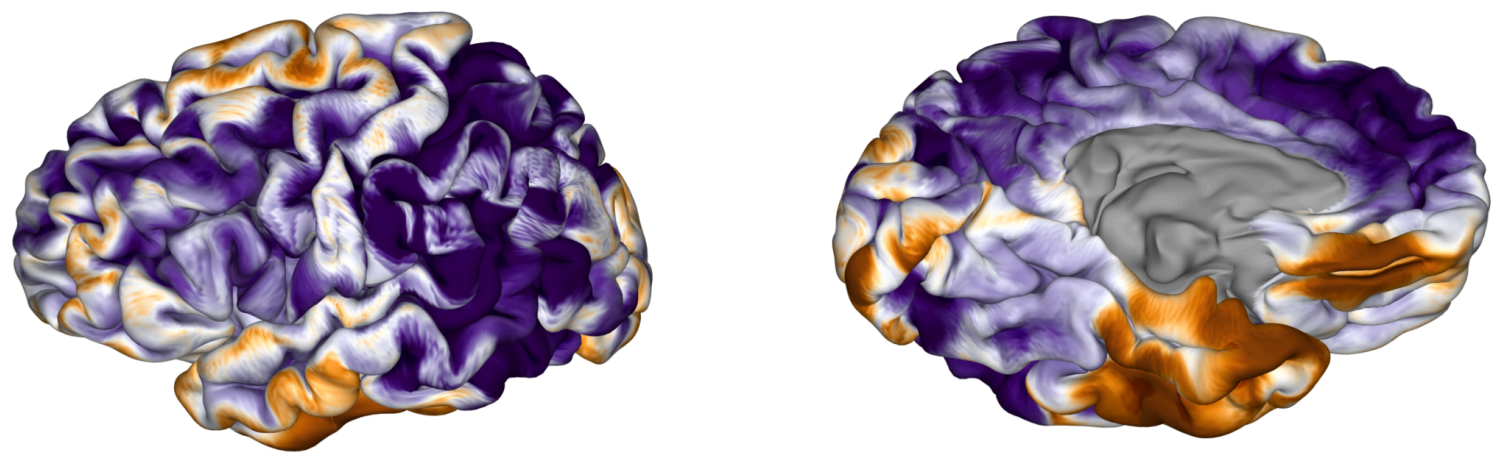

20 100 tSNR

Figure 5. fMRI tSNR. The average tSNR calculated on fMRI scans acquired during a picture naming task shown in the volume (A) and on the cortical surface (B). 
bioRxiv preprint doi: https://doi.org/10.1101/2021.11.18.469030; this version posted November 19,2021. The copyright holder for this preprint (which was not certified by peer review) is the author/funder, who has granted bioRxiv a license to display the preprint in perpetuity. It is made available under aCC-BY-NC-ND 4.0 International license.

A

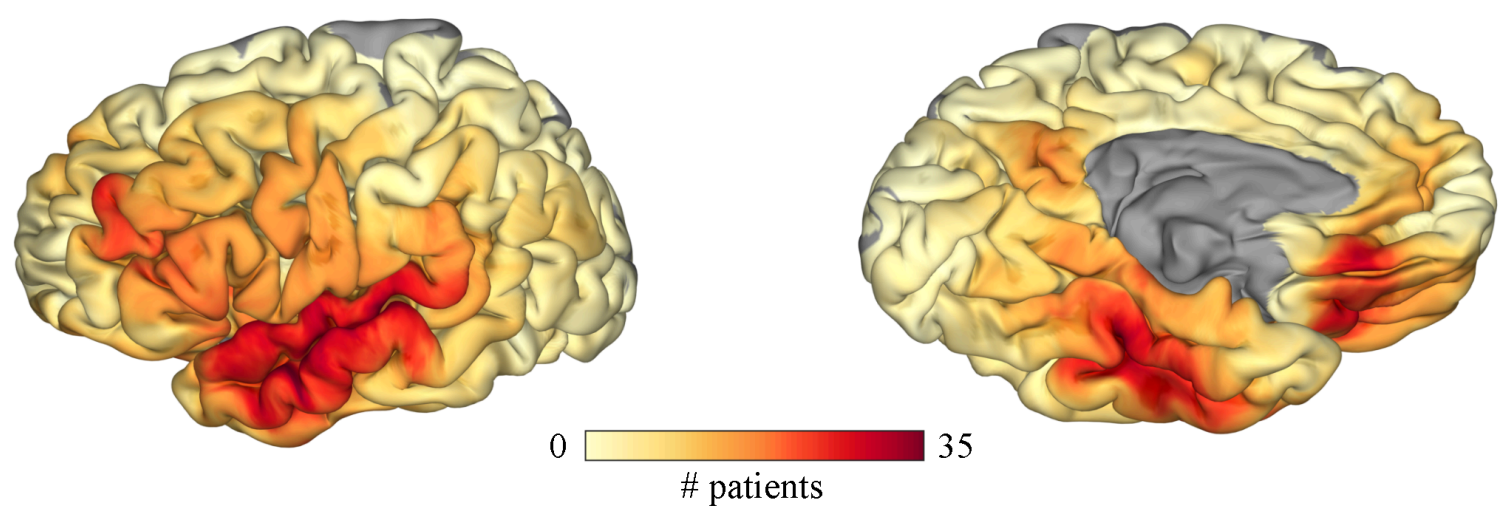

B

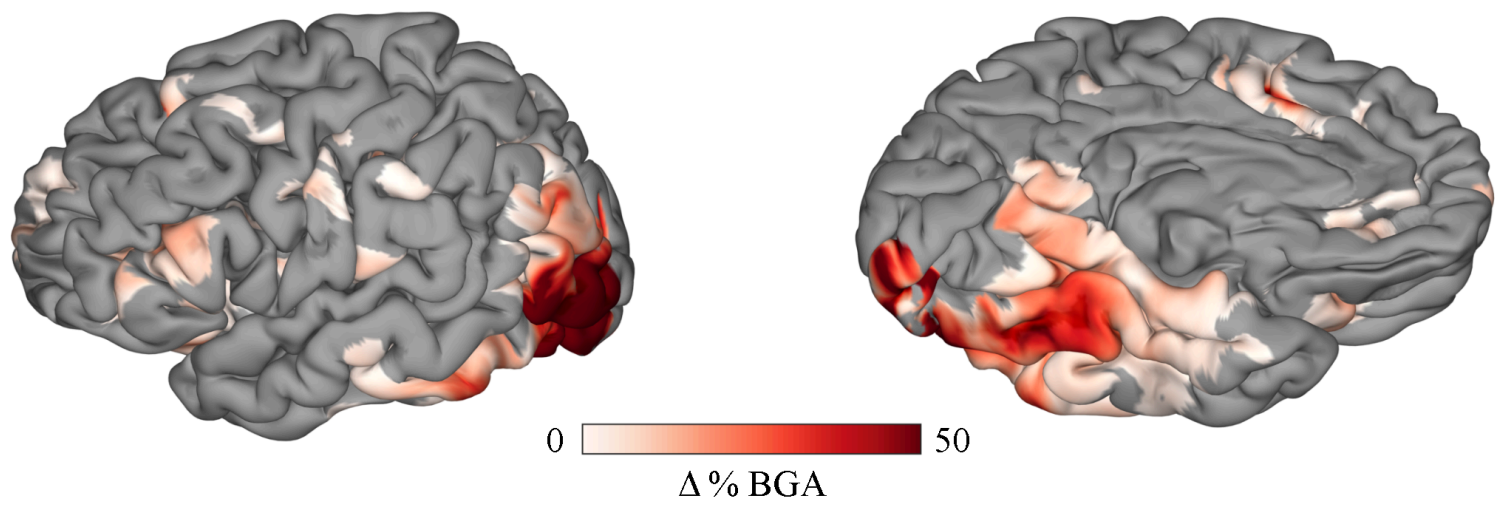

C
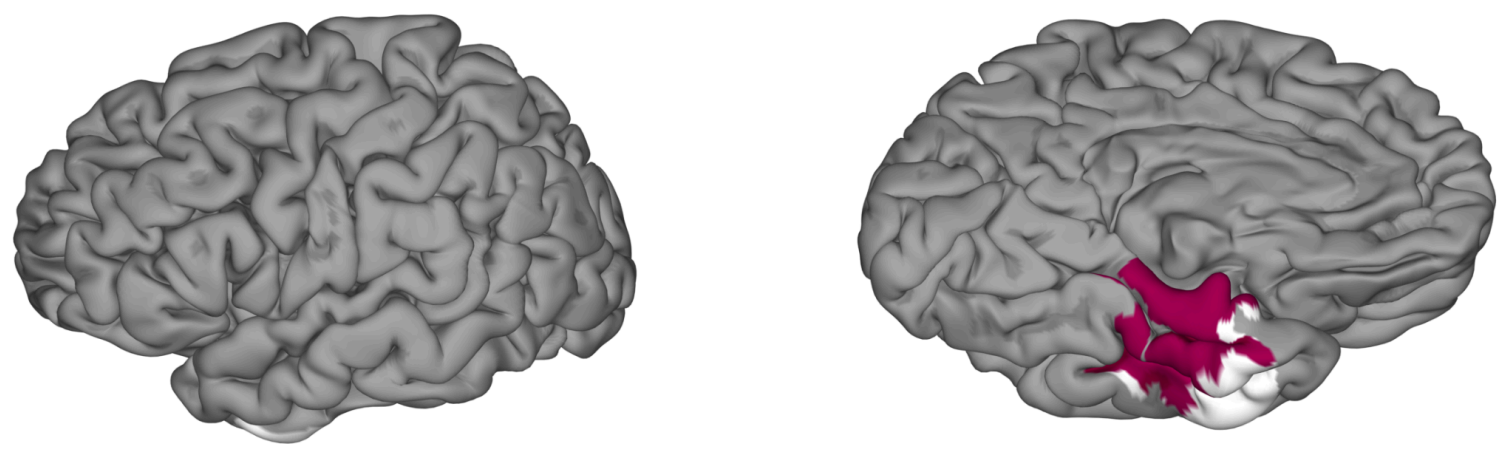

D

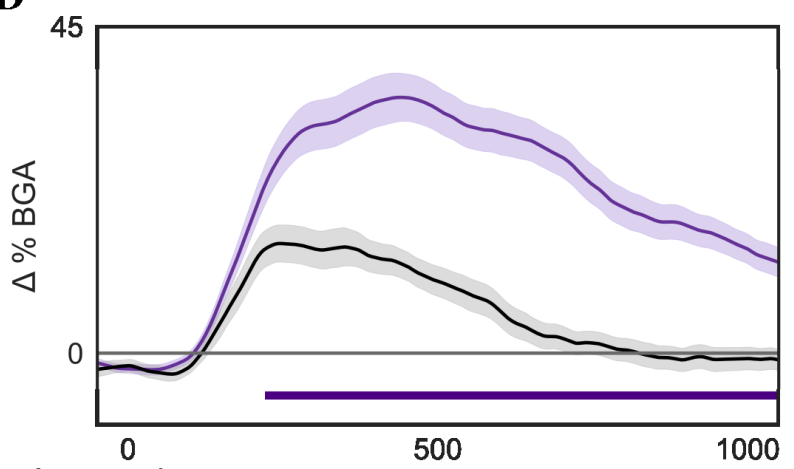

Stimulus Onset

Time (ms)

E

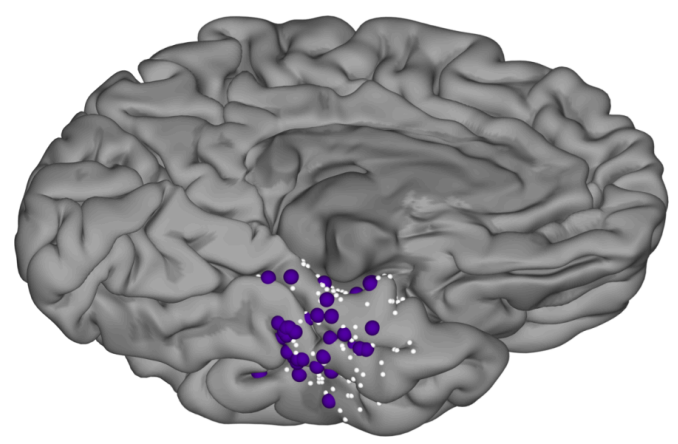


Figure 6. SB-MEMA Results. (A) Coverage of surface recording zones for all left hemisphere electrodes included in the study. (B) Surface-based group-level ECoG estimate of group BGA 500 to $750 \mathrm{~ms}$ following picture onset for pictures of objects $>$ scrambled images. Maps are restricted to regions with significant activity $(\mathrm{p}<0.05$, corrected) and BGA change $>2.5 \%$. (C) Regions in pink represent the overlap between active regions as determined by the thresholded SB-MEMA map and the VLSM significant cluster. Regions in white indicate nodes in the VLSM significant cluster that were not significantly active. (D) Time series average of group estimates of BGA percent change $+/-1$ standard error of the mean following picture onset. Data are smoothed with a Savitsky-Golay filter (third order, $251 \mathrm{~ms}$ length). Significant increase from the control condition (scrambled images) is indicated by the horizontal bar (unpaired t-test, $\mathrm{p}<0.05$, FDR corrected). (D) Electrodes located within the VLSM cluster and SB-MEMA overlap. Electrodes with significant a cortical response included in the average trace are shown in purple, and electrodes that did not have a significant cortical response are shown in white. 